\title{
Triglyceride and Cholesterol Levels in Patients With Chronic Periodontitis
}

\author{
Somaye Ansari Moghadam ${ }^{1}$; Sara Abbasi ${ }^{2,}{ }^{*}$; Esmail Sanei Moghaddam ${ }^{3}$; Alireza Ansari \\ Moghaddam ${ }^{4}$ \\ ${ }^{1}$ Oral and Dental Disease Research Center, Zahedan University of Medical Sciences, Zahedan, IR Iran \\ ${ }^{2}$ Dental Research Center, Zahedan University of Medical Sciences, Zahedan, IR Iran \\ 3 High Institute for Education and Research in Transfusion Medicine, Zahedan, IR Iran \\ ${ }^{4}$ Health Promotion Research Center, Zahedan University of Medical Sciences, Zahedan, IR Iran \\ *Corresponding author: Sara Abbasi, Dental Research Center, Zahedan University of Medical Sciences, Zahedan, IR Iran. Tel: +98-5412446567, E-mail: Abbasi.sara87@yahoo.com
}

Received: May 4, 2014; Revised: February 17, 2015; Accepted: February 25, 2015

\begin{abstract}
Background: Many studies show that periodontitis is a risk factor for serum lipid levels increase. On the other hand, the increase of serum lipids is clearly a risk factor for cardiovascular diseases.

Objectives: The current study aimed to assess the amount of triglycerides and total cholesterol in patients with severe chronic periodontitis. It also evaluated the relationship between the extent of periodontal disease, triglycerides and total cholesterol levels.

Patients and Methods: This case-control study included 61 patients with severe chronic periodontitis and 60 healthy subjects aged 20 - 50 years. The study samples were selected from individuals who referred to periodontics department of dentistry faculty, zahedan university of medical scoences, Iran, from November 2012 to October 2013. The subjects in both groups were matched in terms of age and gender. The extent of periodontal disease, total cholesterol and triglyceride (TG) levels of the subjects were evaluated. Data were analyzed using Pearson Correlation Coefficient and independent sample T-Test.

Results: Triglyceride level in patients with severe chronic periodontitis $(121.67 \pm 67)$ and healthy controls (121.78 \pm 64$)$ was almost the same and the difference was not statistically significant $(\mathrm{P}=0.99)$. Although total cholesterol level was higher in patients with severe chronic periodontitis $(170 \pm 40)$ than the normal subjects $(164.38 \pm 30)$, the difference was not statistically significant $(\mathrm{P}=0.41)$. There was a positive and significant correlation between the extent of periodontal disease and TG level $(\mathrm{P}=0.03)$; while the relationship between the extent of periodontal disease with cholesterol was not statistically significant $(\mathrm{P}=0.4)$.

Conclusions: It seems that cohort studies with larger sample sizes on the relationship between periodontitis and cholesterol level (Total, HDL, LDL) are necessary.
\end{abstract}

Keywords: Chronic Periodontitis; Hyperlipidemias; Triglycerides

\section{Background}

Periodontitis is a common inflammatory disease in humans (1). One of its consequences is the tissue damage as a result of collagen fibers destruction in the periodontal ligaments (2). according to the American Academy of Periodontology if the amount of clinical attachment loss in chronic periodontitis patients is $5 \mathrm{~mm}$ or more, they are considered as patients with severe chronic periodontitis (3). Systemic diseases are one of the risk factors that exacerbate the progress of periodontal disease (4). Hyperlipidemia is one of the diseases considered as a possible risk factor for periodontitis in recent studies $(5,6)$. Nowadays, many people around the world are suffering from hyperlipidemia. Also, the number of patients with hyperlipidemia is increasing in Iran: $23 \%$ of the Iranian adults over 20 years have cholesterol levels higher than normal (7). Total cholesterol includes LDL, HDL and VLDL; the optimal levels of cholesterol and triglyceride are 200 and $150 \mathrm{mg} / \mathrm{dL}$, respectively (8). Endotoxemia, induced by bacterial lipopolysaccharide, can cause periodontitis along with an increase in pro-inflammatory cytokines secretion, which results in an impaired lipid metabolism and the increase of lipid levels. This mechanism can explain the possible association between periodontitis and hyperlipidemia (9). Recent studies on the relationship between periodontitis and hyperlipidemia show that periodontitis is a starting risk factor for hyperlipidemia $(10,11)$. Besides, many studies demonstrated that serum lipid levels were higher in the patients with periodontal disease compared to the healthy individuals, and the presence of periodontitis increases triglycerides and total cholesterol $(6,12)$.

\section{Objectives}

Due to the prevalence of hyperlipidemia and the consequent increase of cardiovascular disease in Iran, the current study aimed to compare the triglycerides serum levels with total cholesterol level in participants with both chronic periodontitis and healthy controls, respectively. Many studies have examined the relationship between severity of chronic periodontitis and hyperlipidemia,

Copyright ( ) 2015, Health Promotion Research Center. This is an open-access article distributed under the terms of the Creative Commons Attribution-NonCommercial 4.0 International License (http://creativecommons.org/licenses/by-nc/4.0/) which permits copy and redistribute the material just in noncommercial usages, provided the original work is properly cited. 
while few have investigated the relationship between the extent of periodontal disease and hyperlipidemia. Therefore, the current study also aimed to examine the relationship between the extent of periodontal disease and serum level of total cholesterol and triglyceride.

\section{Patients and Methods}

The current case-control study was conducted on 121 non-diabetic subjects aged 20 - 50 years from November 2012 to October 2013. Sixty one participants were affected with severe chronic periodontitis and the remaining 60 were healthy regarding periodontal status. Subjects were matched in terms of age and gender. The inclusion criteria for the study were: a) age between 20 and 50; b) no systemic diseases such as diabetes; c) no history of tobacco use; d) no history of anti-inflammatory drugs and antibiotics within the last month. The study was approved by the ethical committee of zahedan university of medical sciences and health services (No.124-92), and the participants signed consent forms.

\subsection{Clinical Assessment}

All of the 60 healthy subjects participating in the study were selected from those who referred to the faculty of dentistry in Zahedan University of Medical Sciences, Iran, (location N: $29^{\circ} 29^{\prime} 46.2 ”$, E: $60^{\circ}$ 51' 1.3"). Clinically, they had no signs of gingival inflammation and no evidence of any germs and calculus. Bleeding on Probing (BOP) was negative for these people and none needed periodontal treatment. In addition, their plaque index was less than $20 \%$. All these people were examined for Clinical Attachment Loss (CAL), Recession (Rec), Probing Depth (PD) and plaque score in six sites (Mesiobuccal, Buccal, Distobuccal, Mesiolingual, Lingual, and Distolingual) on each tooth.

Patients with aggressive periodontitis were excluded based on the criteria such as:

- Family history of aggressive disease.

- Absence of large accumulation of plaque and calculus.

- The rapid rate of disease progression (4).

Those with the following criteria were enrolled as people with severe chronic periodontitis:

- Amount of destruction consistent with local factors.

- Sub-gingival calculus.

- Slow-to- moderate rate of progression.

- Clinical attachment loss $\geq 5 \mathrm{~mm}$ (4).

Moreover, the extent of disease for all subjects was measured according to the following formula:
Extent of periodontal disease $=$ sites with $\mathrm{CAL} \geq 5 \mathrm{~mm} /$ total number of examined dental sites All measurements were carried out using Williams standard probe with a precision of $1 \mathrm{~mm}$, and All measurements were carried out using one examiner.

\subsection{Laboratory Assessment}

In the current study, $4 \mathrm{~mL}$ fasting blood samples were taken from each subject and triglycerides and cholesterol levels were measured using photometric method (Parsazmoon company, 91010, GPO-PAP, Iran).

\subsection{Statistical Analysis}

Data were analyzed using SPSS software, version 20. The association between the extent of periodontal disease, and total cholesterol and TG level were examined by The Pearson correlation coefficient. Additionally, independent sample t-Test was used to compare TG and CHOL levels between the subjects.

\section{Results}

Sixty subjects with severe chronic periodontitis and 61 healthy subjects who participated in the present study were matched in terms of age and gender; thus, each group included 50\% males and 50\% females. Based on the findings (Table 1), TG levels in patients with severe chronic periodontitis $(121.67 \pm 67)$ and the controls (121.78 \pm 64$)$ were almost the same with no statistically significant difference. Also, total cholesterol level in patients with severe chronic periodontitis $(170 \pm 40)$ was higher than those of the subjects of the control group (164.38 \pm 30$)$, but the difference was statistically insignificant.

As shown in Table 2, there was a positive and significant relationship between the extent of periodontal disease and TG levels ( $P=0.03, r=0.3$ ), but the relationship between the extent of periodontal disease and total cholesterol was statistically insignificant $(P=0.4, r=0.1)$.

Table 1. Comparison of Total Cholesterol and Triglyceride Levels Between the Patients and Healthy Control Subjects ${ }^{a, b}$

\begin{tabular}{lccc}
\hline Variables & Patients & Healthy Control & PV \\
\hline Triglyceride & $121.67 \pm 67$ & $121.78 \pm 64$ & 0.99 \\
Cholesterol & $170 \pm 40$ & $164.38 \pm 30$ & 0.41 \\
\hline
\end{tabular}

a Abbreviations: $\mathrm{CHOL}$, cholesterol; TG, triglyceride; PV, P value.

$\mathrm{b}$ Data are presented as mean \pm SD.

Table 2. The Correlation Between Triglyceride, Total Cholesterol and Extent of Periodontal Disease ${ }^{\text {a }}$

\begin{tabular}{lccccc}
\hline Variables & \multicolumn{3}{c}{ Patients } & & Healthy Control \\
\cline { 2 - 6 } & TG & CHOL & Extent & TG & CHOL \\
\hline Triglyceride & - & $r=0.12, P=0.42$ & $r=0.3, P=0.03$ & $r=0.007, \mathrm{P}=0.96$ \\
Cholesterol & $\mathrm{r}=0.12, \mathrm{P}=0.42$ & - & $\mathrm{r}=0.01, \mathrm{P}=0.40$ & $\mathrm{r}=0.007, \mathrm{P}=0.96$ \\
\hline
\end{tabular}

a Abbreviations: CHOL, cholesterol; TG, triglyceride. 


\section{Discussion}

Periodontitis is a gram-negative infection, which leads to severe inflammatory reaction that can result in intravascular release of microorganisms and their production throughout the body $(1,13)$. Also, the increase of Creactive protein levels and serum fibrinogen as a result of periodontitis are accepted as risk factors for cardiovascular diseases (14). In periodontal disease, cytokines secretion increase is observed, which results in impaired fat metabolism and hyperlipidemia. On the other hand, hyperlipidemia is known as a risk factor for cardiovascular diseases $(5,15)$.

The findings of the current study suggest that TG and total CHOL levels, in patients with severe chronic periodontitis were higher than those of the healthy controls, but the difference was statistically insignificant. In Line with these findings, the study conducted by Hamissi et al. (16) in Iran, showed that compared to healthy subjects the increase of TG and cholesterol levels in subjects with periodontitis was statistically insignificant. Similarly, many other studies have reported that total CHOL and TG levels in patient with chronic periodontitis were significantly higher than those of the healthy subjects $(12,17)$. Regarding the fact that the influence of age on chronic systemic disease is proved, these significant results may be due to higher age average of patients with periodontitis (50) compared to that of the healthy controls (41) in these studies (18). Losche et al. obtained similar result in a study which had the shortcoming of the previous studies (6).

Previous studies conducted in Iran by Taleghani et al. (17) and Moein Taghavi et al. (12) reported the increase of TG and total CHOL levels in people with chronic periodontitis, in comparison to the healthy subjects, as significant. In addition, the current study was a cross-sectional study with a modest sample size, which can partly justify the significant difference of the former Iranian data with those of the present study. Another finding of the current study was the positive and significant correlation of TG levels with the extent of periodontal disease; in other words, increase of the periodontal disease extent lead to increase in TG levels.

Moreover, no association was observed between cholesterol level and the extent of periodontal disease in the current study. Recent studies are indicative of a positive relationship between hyperlipidemia and severe chronic periodontitis. However, this relationship was not observed between the mild form of periodontal disease as well as the risk of hyperlipidemia in patients with severe chronic periodontitis; risk of hyperlipidemia was 30\% higher than those of the normal subjects $(19,20)$. Observations of Sridhar et al. indicated that TG levels were not correlated with attachment loss increase in patients with periodontitis (5).

Many studies have already investigated the relationship between hyperlipidemia and periodontal disease $(12,16$,
21), but studies on the association between the extent of periodontal disease and hyperlipidemia are few. The current study, as one of the few, carried out in this area, can cast light on future researches and contribute to finding the unknown cause of the relationship between these two diseases.

In future studies, it is recommended to use LDL and HDL as complementary tests along with the measurements of total cholesterol to obtain more complete results. Also, the current study was a cross sectional study with a more modest sample size and cannot be used to determine the causal relationship. Thus, cohort studies with large sample sizes should be conducted to obtain comprehensive information.

\section{Acknowledgements}

Authors are grateful to the staff of Periodontology Department of Zahedan Faculty of Dentistry, Zahedan Blood Transfusion Organization, Research Institute for Health Promotion, and Zahedan University of Medical Sciences and Health Services, Iran, for their kind cooperation in the current investigation.

\section{Authors' Contributions}

Somaye Ansari Moghadam and Sara Abbasi: data research and manuscript writing. Alireza Ansari Moghaddam: data analysis and manuscript revising. Esmail Sanei Moghaddam: performing laboratory tests and manuscript preparation.

\section{References}

1. Pihlstrom BL, Michalowicz BS, Johnson NW. Periodontal diseases. Lancet. 2005;366(9499):1809-20.

2. Preshaw PM, Alba AL, Herrera D, Jepsen S, Konstantinidis A, Makrilakis K, et al. Periodontitis and diabetes: a two-way relationship. Diabetologia. 2012;55(1):21-31.

3. Savage A, Eaton KA, Moles DR, Needleman I. A systematic review of definitions of periodontitis and methods that have been used to identify this disease. J Clin Periodontol. 2009;36(6):458-67.

4. Michael GN, Henry T, Perry RK, Carranza FA. Carranza's clinical eriodontology. 11th edCalifornia. Mosby: Elsevier Health Sciences; 2011.

5. Sridhar R, Byakod G, Pudakalkatti P, Patil R. A study to evaluate the relationship between periodontitis, cardiovascular disease and serum lipid levels. Int J Dent Hyg. 2009;7(2):144-50.

6. Losche W, Karapetow F, Pohl A, Pohl C, Kocher T. Plasma lipid and blood glucose levels in patients with destructive periodontal disease. JClin Periodontol. 2000;27(8):537-41.

7. Azizi F, Rahmani M, Ghanbarian A, Emami H, Salehi P, Mirmiran $\mathrm{P}$, et al. Serum lipid levels in an Iranian adults population: Tehran Lipid and Glucose Study. EurJ Epidemiol. 2003;18(4):311-9.

8. American Heart Association.. Copyright Notice. 2014. Available from: http://www.heart.org/HEARTORG/General/Copyright-Notice_UCM_300378_Article.jsp.

9. Iacopino AM. Periodontitis and diabetes interrelationships: role of inflammation. Ann Periodontol. 2001;6(1):125-37.

10. Tomofuji T, Kusano H, Azuma T, Ekuni D, Yamamoto T, Watanabe T. Effects of a high-cholesterol diet on cell behavior in rat periodontitis.J Dent Res. 2005;84(8):752-6.

11. Marjanovic M, Buhlin K. Periodontal and systemic diseases among Swedish dental school patients - a retrospective register study. Oral Health Prev Dent. 2013;11(1):49-55. 
12. Moein Taghavi AM, Haerian Ardakani A, Talebi Ardakani MR, Tabatabai I. Evaluation of association between hyperlipidemia and periodontitis.J Dental Med. 2005;18(2):53-8.

13. Geerts SO, Nys M, De MP, Charpentier J, Albert A, Legrand V, et al. Systemic release of endotoxins induced by gentle mastication: association with periodontitis severity. J Periodontol. 2002;73(1):73-8.

14. Ridker PM, Rifai N, Rose L, Buring JE, Cook NR. Comparison of C-reactive protein and low-density lipoprotein cholesterol levels in the prediction of first cardiovascular events. $N$ Engl J Med. 2002;347(20):1557-65.

15. Lowe GD. pathogenesis of cardiovascular disease. Ann Periodontol. 1998;3(2):12-5.

16. Hamissi J, Shahsavarani MT, Hamissi H. A Comparison of Serum Lipid Profile between Periodontitis Patients and Healthy Individuals. Iran Red Crescent Med J. 2011;13(4):283-4.
17. Taleghani F, Shamaei M, Shamaei M. Association between chronic periodontitis and serum lipid levels. Acta Med Iran. 2010;48(1):47-50.

18. Cutler CW, Shinedling EA, Nunn M, Jotwani R, Kim BO, Nares S, et al. Association between periodontitis and hyperlipidemia: cause or effect? J Periodontol.1999;70(12):1429-34.

19. Mattila KJ, Valtonen VV, Nieminen M, Huttunen JK. Dental infection and the risk of new coronary events: prospective study of patients with documented coronary artery disease. Clin Infect Dis. 1995;20(3):588-92.

20. Katz J, Chaushu G, Sharabi Y. On the association between hypercholesterolemia, cardiovascular disease and severe periodontal disease. J Clin Periodontol. 2001;28(9):865-8.

21. Machado AC, Quirino MR, Nascimento LF. Relation between chronic periodontal disease and plasmatic levels of triglycerides, total cholesterol and fractions. Braz Oral Res. 2005;19(4):284-9. 